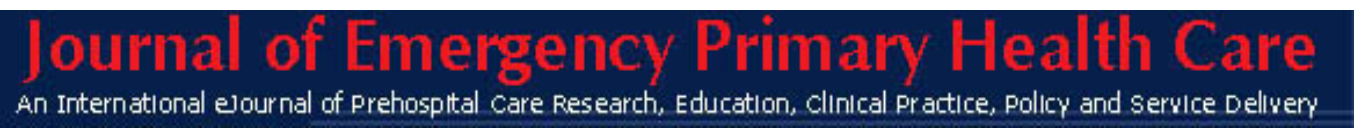

ISSN 1447-4999

\author{
Policy and Service Delivery
}

\title{
RIGHT TECHNOLOGY, RIGHT SITUATION - A CASE REPORT ON PREHOSPITAL TELEMEDICINE \\ Article No. 990020
}

Brian Meade, BSC (Hons), PhD - Associate Fellow, Faculty of Accident and Emergency Medicine Royal College of Physicians and Surgeons, UK.

Peter Barnett, MBBS, MSc(Epid). FRACP, FACEM - Deputy Director of the Department of Emergency Medicine, Royal Children's Hospital, Melbourne.

Tony Walker, BParamedStud, MICACert, GDipEd, GCertAppMgt, MACAP - Manager, Clinical and Education Services, Rural Ambulance Victoria.

Keywords: EMS; informatics; satellite communications; telemedicine; telecommunications

Telemedicine can be conducted through a range of media including phone, facsimile, email, Internet, or by fixed, satellite or microwave video-conferencing. Interestingly the technology does not always need to be complicated, as demonstrated by the first recorded instance of telemedicine, which occurred when Morse Code was used from an Antarctic base to the Australian mainland for medical advice. Nowadays NASA uses extremely sophisticated telemetry systems to monitor the health of astronauts, since it would cost many millions of dollars to evacuate a sick member to earth.

The Internet can be used for videoconferencing, but it has a propensity to be an unstable platform. Microwave \& other radio carriers offer fast connection but require line-of-sight (direct visual sight with the receiving end) which can be problematic in many rural areas. There is a strong move towards 'broadband' for videoconferencing, but the roll-out of this technology in rural areas is a considerable way off, as is wireless or "bluetooth" technology.

The system currently being piloted in an isolated rural community in South Western Victoria utilizes a combination of satellite Integrated Services Digital Network (ISDN) and videophone terminals, together with various File Transfer Protocols (FTPs) and other networking facilities. This pilot is also trialling a low-cost videophone system, which works across ordinary phone lines, should they be available near the emergency. This offers quite useful low-end colour video.

In terms of visual data, it is usually taken that 128,000 bits per second (128Kbps) is the minimum for satisfactory videoconferencing. Even at this speed of connection much movement causes 'pixellation' or breaking up of the picture. Although the ISDN system used in South Western Victoria nominally works at 64Kbps (about twice the speed of normal or PSTN telephone lines), quite satisfactory video can be achieved. Since movement is not generally important in patient presentation, this means that the remote clinician can have a real-time view of the mechanism of injury and the patient (fractures, pupils and so on) of about the same quality as that offered by polaroid photography (Fig 1). 


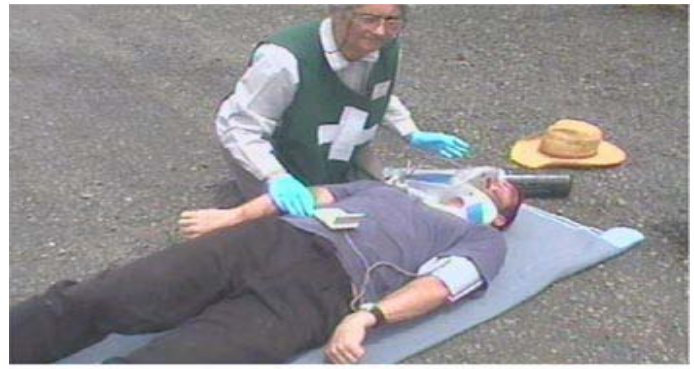

Fig 1 View seen by remote consultant after image has been relayed via an Inmarsat link.

The portable videoconferencing system we use involves a Capsat Messenger TT-308 satellite phone. This is a laptop-sized unit, which offers a portable ISDN connection when interfaced with the Inmarsat satellite network. The particular geostationary satellite we access is $36,000 \mathrm{~km}$ above Fiji. A videoconferencing phone (we use a Motion Media model 225) is connected to the Capsat Messenger, enabling transmission and reception of real-time video to and from any ISDN videoconferencing unit anywhere in the world. There is a slight lag between sound and picture due to the great distance the signal has to travel, but this lag has no clinical significance. The videophone has a 'freeze-frame' mode which enhances the picture considerably.

Since the angle of elevation of the satellite at our latitude is about $40^{\circ}$, in some situations it is difficult to achieve a satisfactory signal - as for example down a gully. In such cases we video the scene, return to a better signal position and re-play the video for the clinician - this is generally represents a delay of only a few minutes. The clinician may then communicate with personnel at the site by radio.

A Telstra 'bridge' is available whereby two other clinicians in different locations may be called for consultation. They may enter the videoconference via a split-screen on the (remote) videophone all in real time.

If there is a normal phone line available, we have found that a low cost PSTN video-phone can transmit very useful information to the clinician. The speed of connection normally being of the order of 24Kbits per second, pixellation would be expected with very much movement. The Motion Media CareStation 125, however, enables the operator to control the frame rate to adjust balance between smoothness (motion) and clarity (detail). As with the satellite phone a freeze function is available (Fig 2).

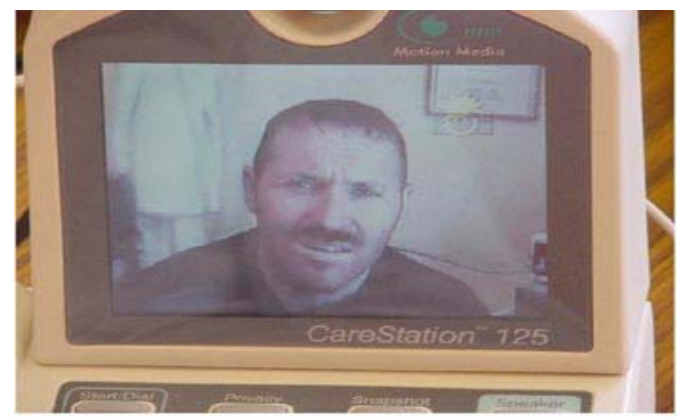

Fig 2 View seen by remote consultant after image has been relayed by ordinary phone line

Both of the above video-phones have ports for other devices such as a document camera or a high quality video camera (which gives a better picture than the small built-in-camera). A 
'video-out' port enables the video to be played onto a larger monitor. Other clinical peripheral devices, which can be plugged in via an RS232 port, include 'vital signs' monitors, digital stethoscopes, otoscope, spirometer, ECG etc.

It is now necessary to evaluate the system to see whether it can help to reduce mortality and morbidity in remote areas.

This article was peer reviewed for the Journal of Emergency Primary Health Care Vol. 1 (1-2) 2003 